\title{
SYSTEM FOR EARTHQUAKE PREDICTION RESEARCH IN THE REGION OF VHF FREQUENCY BAND
}

\author{
Isao Yamamoto, Kiyoshi Kuga, Tohru Okabayashi and Takashi Azakami \\ Department of Information \& Computer Engineering, Okayama University of Science, \\ 1-1 Ridai-cho, 700-0005 Okayama, Japan
}

\begin{abstract}
We propose a system for earthquake prediction research in the region of -VHF frequency band. The system allows us to observe the sporadic radiated electromagnetic wave noises and the pre-seismic phenomena of radio waves.
\end{abstract}

Keywords: Earthquake prediction, FM band, electromagnetic wave noise

\section{Introduction}

Recently, the earthquake activity has been becoming enhanced in the western area of Japan; for example, the Tottori-Seibu earthquake (October 6, 2000, M7.3) and the Heisei 13th year-Geiyo earthquake (March 24, 2001, M6.4). Therefore, there are high demands for research and establishment of a system for earthquake prediction.

It is assumed that earthquakes are generally caused by plate rock in upper side of the mantle crashing in fragments and/or when magma is bursting out into the air from the magmaoverdue. Whatever the case may be, prior to actuation of an earthquake the minerals will break in the form of a comparatively small part. There are many evidences indicating that in a height range from few hundreds to few thousands meters above the epicenter, electrical discharges occur in the atmosphere before an eathquake [1]. Such discharges are the main reason for drastically worsened FM wave propagation and enhanced wave noises. According to the reference [1], the electric charged aerosol will stray for a short period of time at a few kilometers height in the atmosphere. We consider that the electromagnetic waves will be disturbed by the electric charged aerosol. Additionally, we consider that the ionospheric channel is made up by the air shower of the cosmic rays. Furthermore we suspect that with the appearance of those sporadic electromagnetic waves, electric charged aerosols, and ionospheric channels, in a height of few thousands meters we expect that an ionization layer emerges in a short period of time. We call this layer an Epicenter Quick-Setting Ionization (EQSI) layer, and suspect that its occurrence has an important role in earthquake prediction. This EQSI layer will cause radio noise in the electromagnetic wave band.

In this paper, we describe a measuring system for the electromagnetic wave noise prior to an earthquake. As a matter of course, the important items of this prediction are the epicenter of the earthquake, its magnitude, and its estimate time of occurrence. The system allows us to estimate these items almost without delay using the FM band noise of the frequencies same as other literatures [5-8] which deal with the same topic.

\section{Measuring system}

To measure the natural radiation noise of electromagnetic waves before the earthquake occurring, we designed a measuring system as shown in Fig. 1. When an electromagnetic wave noise occurs before an earthquake, the same noise affects the whole FM band, i.e. the same noise can be detected through a booster and a splitter in the lower frequency range and higher frequency range, as well. To filter out sporadic noises not connected to earthquake occurrence, the measurements were performed synchronously in the low and the high parts of the FM band. To detect the direction of locality of an earthquake, six Yagi-Uda antennas pointing in six directions are used. An array of twelve FM tuners (frequency ranging from 76.0 to $108.0 \mathrm{MHz}$ ) was used. To avoid the effect of the radio signals from the FM radio stations, the measurement frequencies were chosen to be as far as possible from the radio emissions. The signals obtained through the FM tuners were converted from analog to digital by digital multi meter (DMM), and were recorded by a personal computer. The system has 


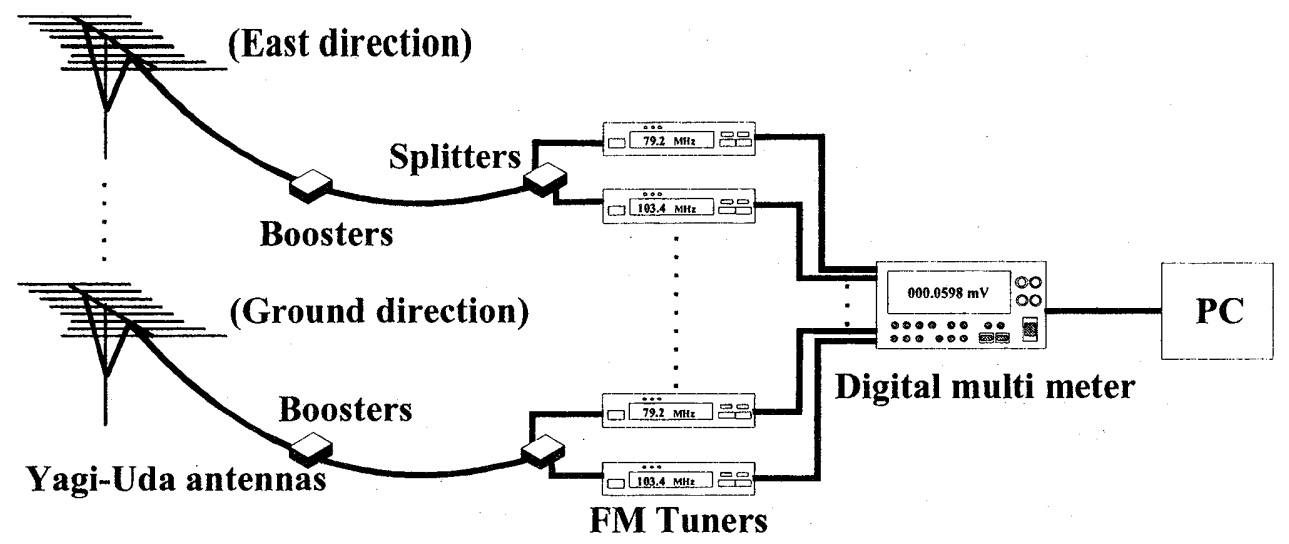

Fig. 1 Schematic diagram of the measuring system for the observation of seismic signals

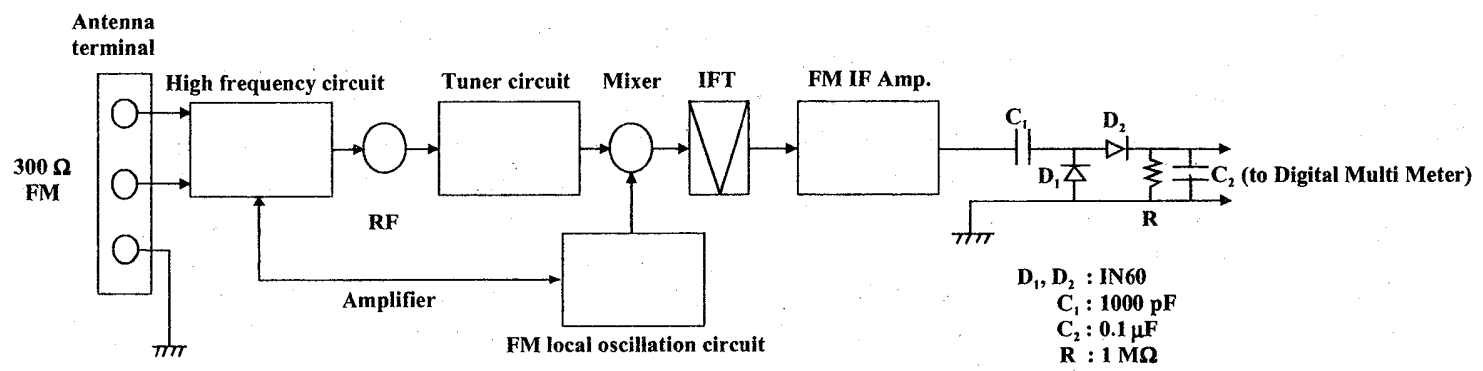

Fig. 2 Detector circuit of the Seismic Electric Signal (SES) receiver.

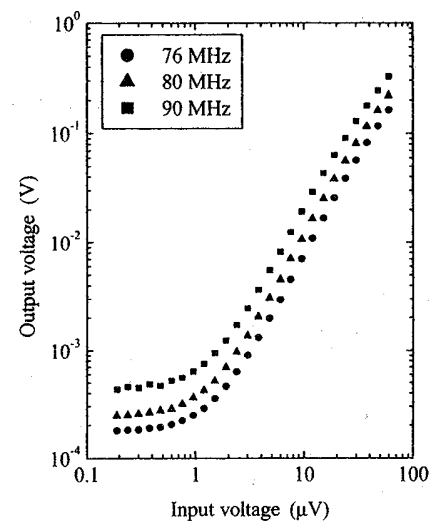

Fig. 3 Input-output characteristics of the detector. 
measuring period of 10 seconds. The intensity of the electric waves from the center of galaxy using an extra antenna system and the temperature in the DMM booth were also recorded by the same DMM. The detector circuit of the Seismic Electric Signal (SES) receivers is shown in Fig. 2. Figure 3 shows the detection characteristics of FM tuner, and Fig. 4 shows filter characteristics of the FM tuner.

The fourteen input channels (for example: 2 East, 2 West, 2 South, 2 North, 2 Heaven, 2 Ground, 1 Galaxy and 1 Temperature) of the DMM (Model 7702, KEITHLEY) are multiplexed every $500 \mathrm{~ms}$ as shown in Fig. 5. To avoid the switching noise the readings are performed $400 \mathrm{~ms}$ after the channel change. The sampling period is $10 \mathrm{~s}$.

Figure 6 shows a radio noise from an antenna that is focusing to the center of galaxy $\left(26^{\circ}\right.$ of elevation angle, southern direction) at the Okayama Ridai Seismic Observation Center (ORSOC; Director Prof. Dr. Isao Yamamoto) that is located geographically at E $133^{\circ} 55^{\prime} 48.3^{\prime \prime}, \mathrm{N} 34^{\circ} 41^{\prime} 48.1^{\prime \prime}$ and an altitude of 70 meters in Okayama City.

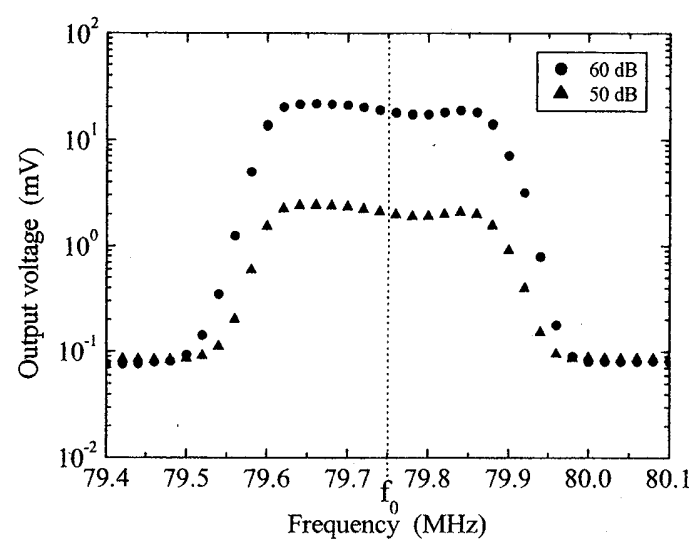

Fig. 4 Receiving bandwidth of tuner characteristics.

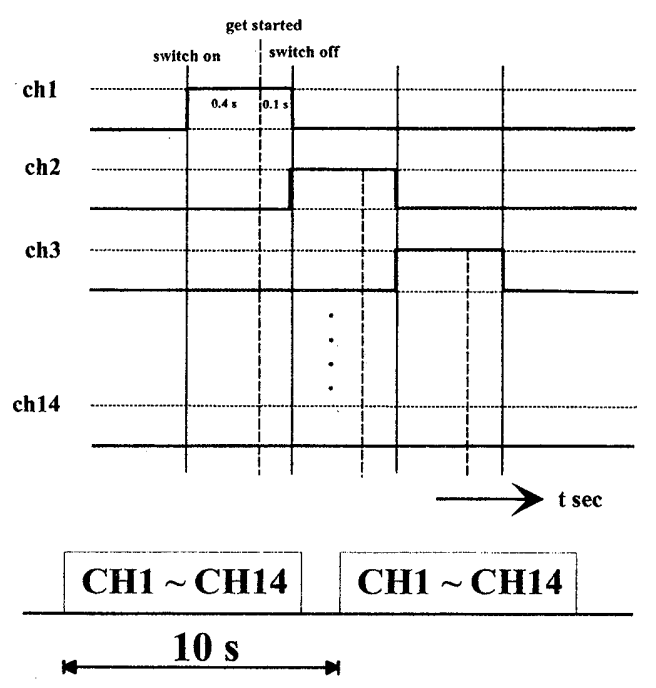

Fig. 5 Channel switching diagram of digital multi meter (DMM).

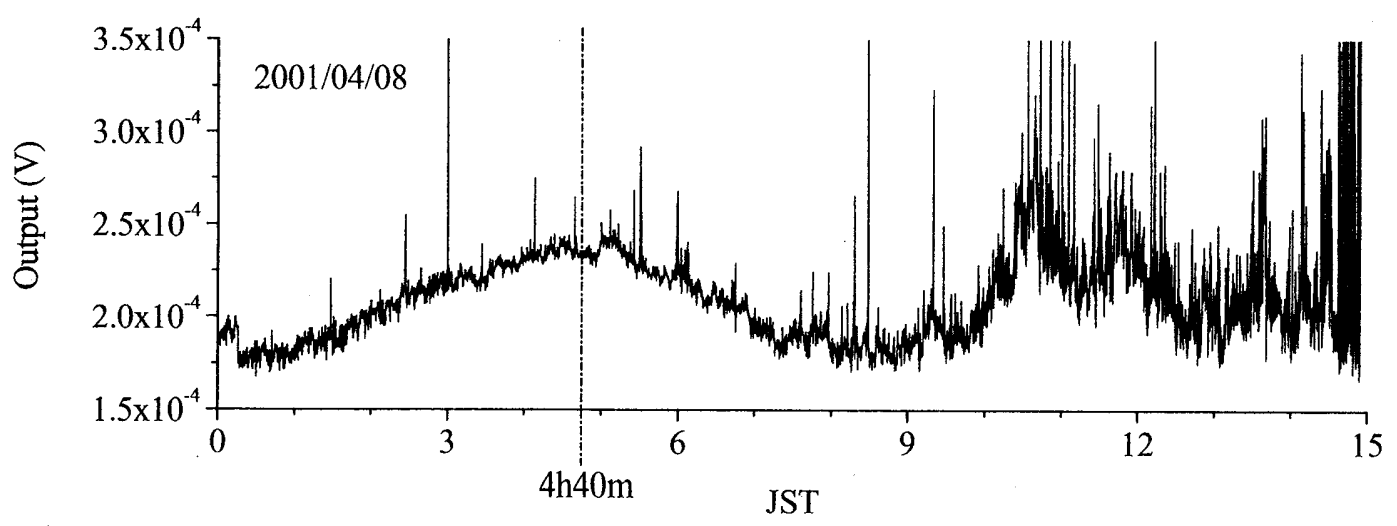

Fig. 6 Electromagnetic waves received from the center of galaxy $\left(17^{\mathrm{h}} 45.7^{\mathrm{m}}\right.$ of inclination and $-29^{\circ}$ $00^{\prime}$ of declination) at an elevation angle $26.306^{\circ} . \mathrm{f}=79.75 \mathrm{MHz}$. 
According to the Chronological Scientific Table 2001, the center of galaxy crosses the meridian as of $4^{\mathrm{h}} 40^{\mathrm{m}}$ hours, April 8, 2001. It can be seen from Fig. 6 that the peak value of the measurement data is approximately at that time. Therefore, this apparatus can also be used to monitor the intensity of the radio waves from the center of galaxy.

\section{Miscellaneous measurement results}

The typical measurement data at the ORSOC for the interval from the beginning of January 2001 up to the present, is explained below.

\subsection{The 2001 Geiyo Earthquake (March 24, 2001)}

Channel 3 of the SES measurements on the day of 2001 Geiyo Earthquake seems to indicate the premonitory symptoms of an earthquake in Fig. 7 [2]. $94.75 \mathrm{MHz}$ of frequency and two-divided splitters were used between $2001 / 3 / 24$ to $3 / 2520: 44$. Also, $79.75 \mathrm{MHz}$ of frequency and twelve-divided splitters were used after $3 / 25$ 20:44. According to the Meteorological Agency of Japan, the depth of the seismic center is $60 \mathrm{~km}$, and the magnitude is 6.4 . The distance from here to the epicenter is $140 \mathrm{~km}$.

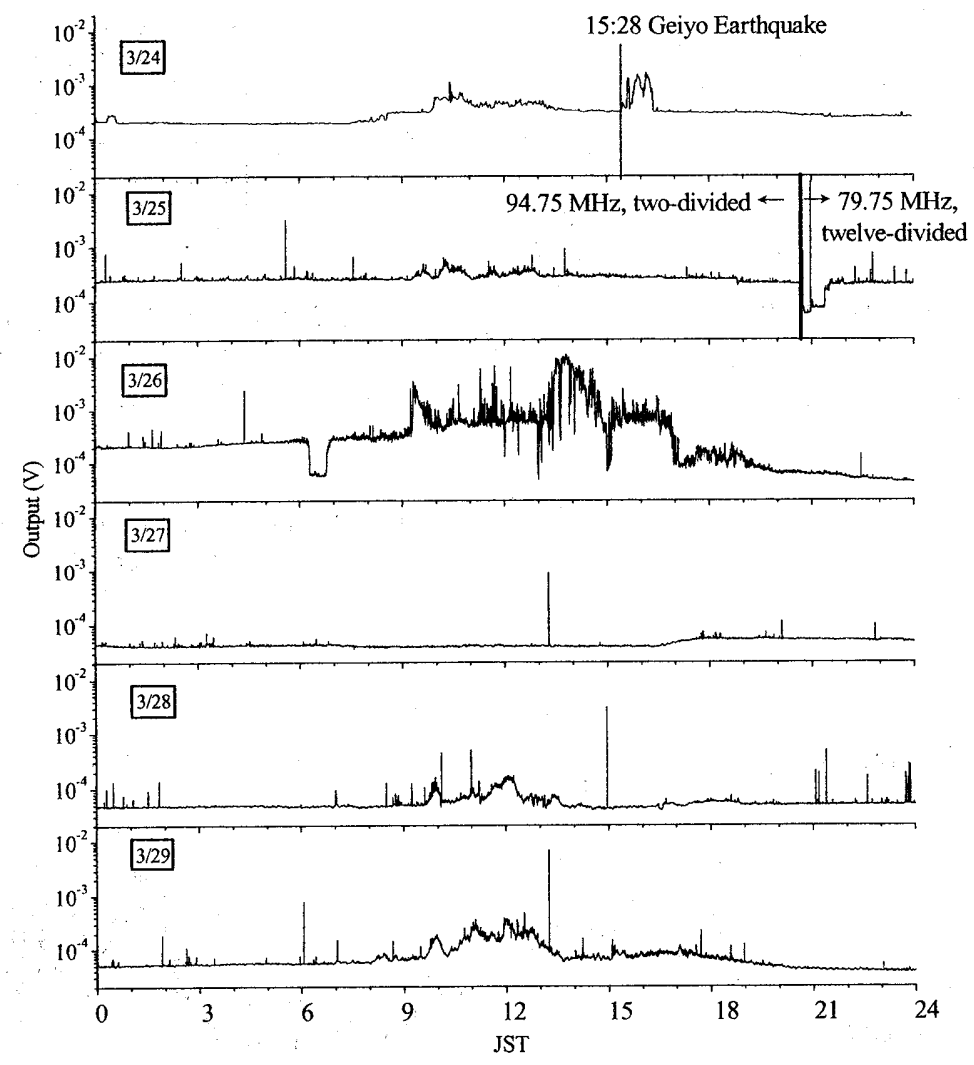

Fig. 7 SES data for the 2001 Geiyo Earthquake. 


\subsection{Electromagnetic noise of atmospherics (July 17, 2001)}

Figure. 8 shows the electromagnetic wave noise of atmospherics at the ORSOC and its neighborhood on July 17, 2001. By visual observation the occurrences were as follows, 0942TL(NW)-0947TL(Z)-1000TL(NE)-1240TL(W)-1305TL(NW)-1330T(NW)-1355, where the letters $\mathrm{T}$ and $\mathrm{L}$ mean thunderbolt and lightning, respectively [3]. For comparison the signal of thunderbolt recorded by our apparatus is shown in Fig. 8. Such signals were often recorded and confirmed through the whole summer season.

\subsection{The observation of the Leonis meteor swarm (November 19, 2001)}

To classify the electromagnetic wave related with the precursor of earthquakes without other noise sources such as meteor, the authors observed the radio noise caused by the famous Leonis meteor swarm. Figure 9 shows measured data on the noise of electromagnetic waves caused by the Leonis meteors swarm. Data of channel 5 in Fig. 9 were recorded by a Heaven direction antenna. The number of meteors can be found from the data pulses (threshold of $5 \times 10^{-5} \mathrm{~V}$ obtained by differentiation of channel 5 in Fig. 9). Figure 10 shows the occurrence histogram of the pulse number in 30 minutes intervals on November 19 (the Leonis meteor swarm day) and the average of pulse number between November 1 and 18. The peak time at 4:00 AM (JST) of the Leonis meteor swarm day is exactly the same as it was announced by the mass media [4].
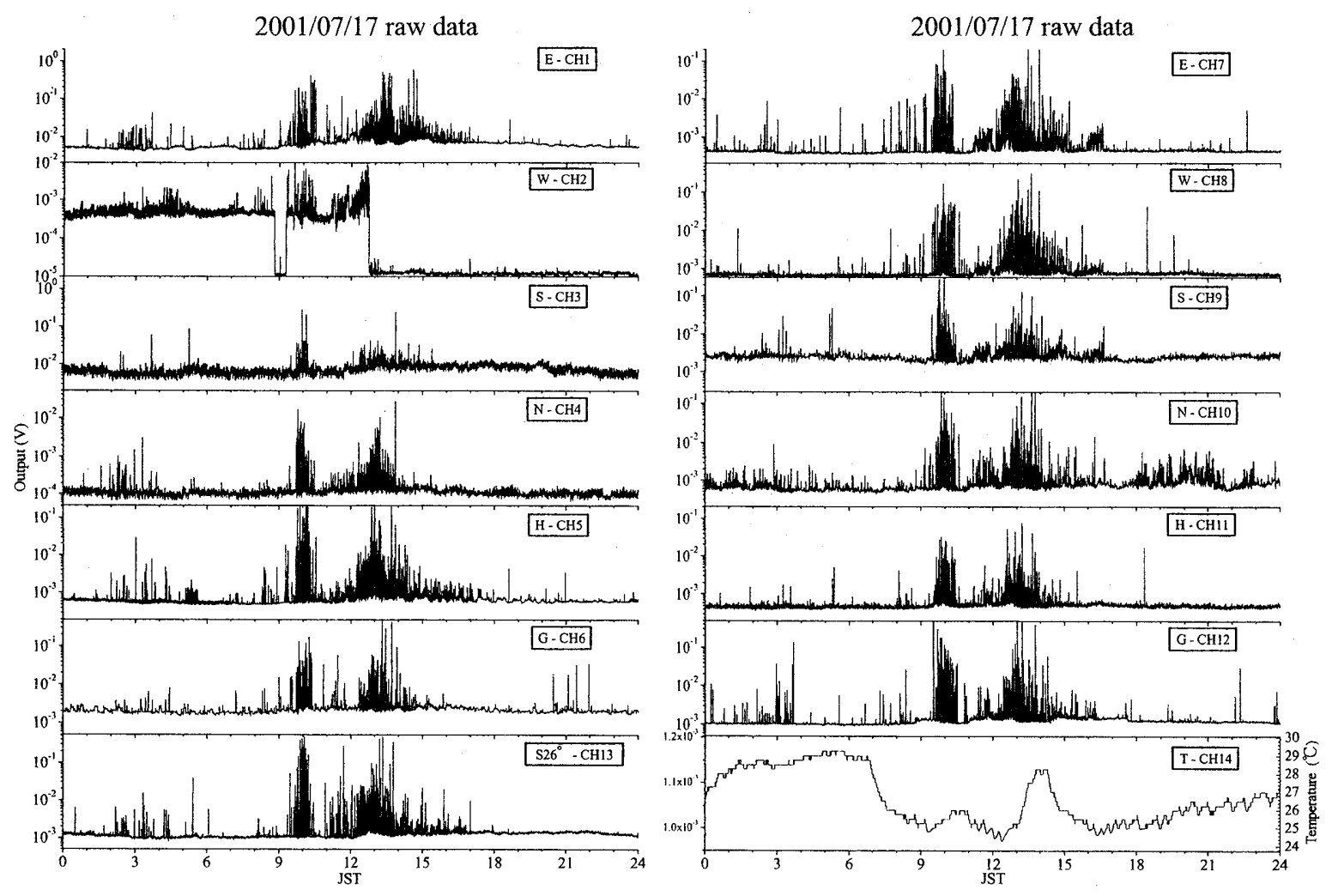

Fig. 8 Electromagnetic wave noise of the atmospherics at the ORSOC and its neighborhood. Frequencies $79.75,77.85,77.85,79.75,79.75,79.75,101.35,101.45,101.35,100.90,102.20$, $101.35,79.75 \mathrm{MHz}$ for channels 1 to 13 , respectively. 


\section{Discussion}

Various techniques for earthquake prediction like microscopic anomaly [10], ion gaseous aerosol [1], electromagnetic waves [9], borehole volume strain meter [11], etc. were reported in the last years.

In [9] a method for prediction of earthquakes based on the measurement of electromagnetic noises is shown, but it is important to predict also where the epicenter of the predicted earthquake will be. From this point of view, the electromagnetic wave method proposed here has an advantage to predict the epicenter using vector field techniques, and in addition, compared to the methods proposed in [1] and [10], the number of observation sites is reduced to one.

Furthermore, the electromagnetic wave techniques in the FM frequency band have some other advantages, particularly: (1) easy to handle the system, (2) economical. Because the observation apparata can be obtained from the market. When we try to predict the epicenter of an earthquake it is necessary to distinguish explicitly the electromagnetic wave noise caused by the earthquake from any other electromagnetic wave noises (for example, the sun, urban, meteoritic, a flash of lightning, etc.). To distinguish the different noise sources like meteor and flash of lightnings etc, we analyze the measurement data from two different frequencies in the FM band obtained synchronously.

The proposed system is shown in Fig. 1. Using commercial FM receivers the measurements are performed in two different frequencies. Generally, around the observation site there are many FM station broadcasts using their respective frequency bands. To obtain only the electromagnetic wave noise, it is necessary to receive the FM signal through a suitable narrow band filter. The filter characteristics are shown in Fig. 4. The filter has a throughput band of $\pm 150 \mathrm{kHz}$, and is sufficient to select a measurement signal among the many broadcast stations. This equipment with input-output characteristics as shown in Fig. 3, is able to detect signals with very low voltage level $(0.2 \mu \mathrm{V})$. The 14 sets of tuners work in pairs successively with a sampling interval of 10 seconds as shown in Fig. 5 .
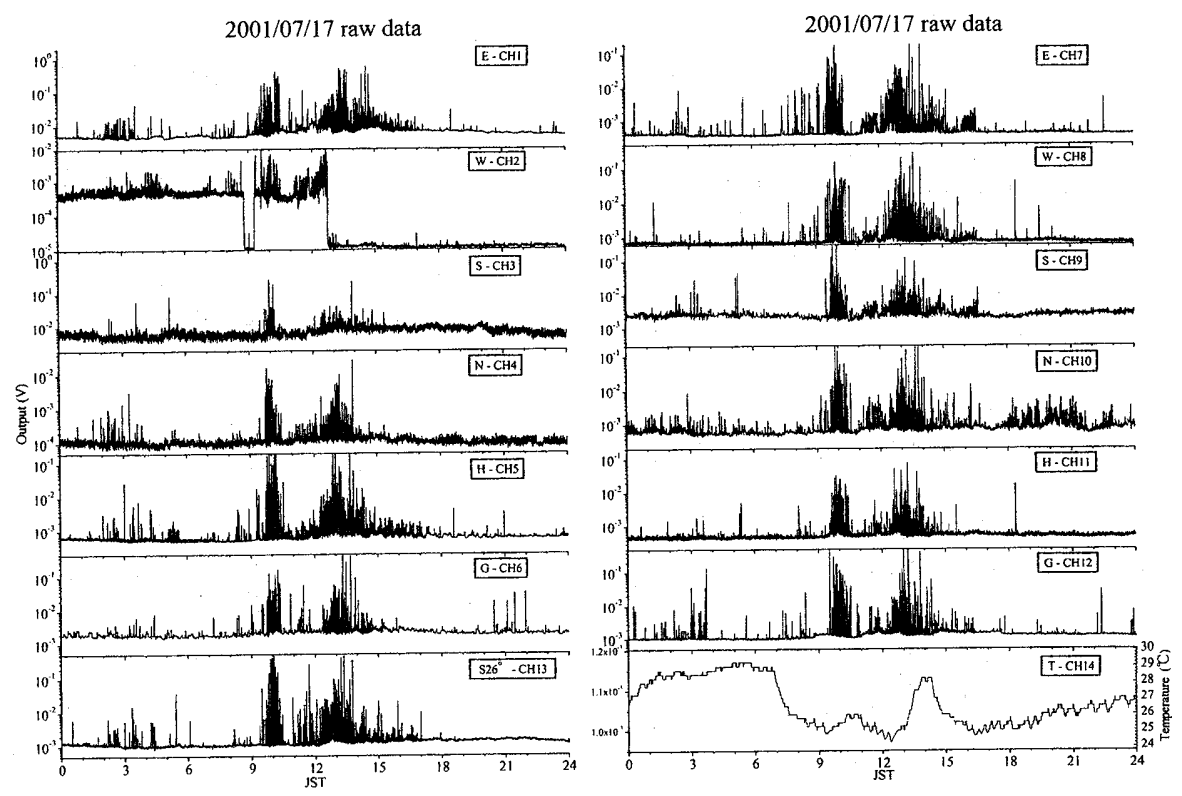

Fig. 9 Noise intensity of the electromagnetic waves by Leonis meteor swarm. Frequencies 79.75, $77.80,77.85,79.75,79.75,79.75,101.35,101.45,101.35,100.90,102.20,101.35,79.75 \mathrm{MHz}$ for channels 1 to 13 , respectively. 
Figure 6 is an example for performance of the equipment. This measurement data presents the intensity of an electromagnetic wave received from the center of the Galactic system using a 10-elements Yagi-Uda antenna which is set at an angle of elevation $26^{\circ}$ in south direction. At the time of measurement the center of the Galactic system crossed the meridian at 4:40 JST. Noise fluctuations of $0.55 \times 10^{-4} \mathrm{~V}$ can be clearly distinguished from the data taken 3 hours before and after the crossing of the meridian. These noise fluctuations are almost equal to the sensitivity limitations of the observation apparatus (see Fig. 3). Since, in order to achieve an earthquake prediction, there is a need to make accurate distinction between the different noises as mentioned above. It should be noted, however, that noise from the center of the Galactic system does not appear seldom in cloud weather or at the summer season. As the intensity of radio wave signals from the Galaxy is extremely weak and is almost in the vicinity of the limitations for the measuring apparatus, it is necessary to investigate the space environmental situation quantitatively (for example, at time of thick clouds etc.), in order to develop the right reasoning about the patterns composed with background noises.

Another example for well-known electromagnetic noise sources is lightning. Figure 8 shows a result of observation on July $17 \mathrm{th}, 2001$ when there was heavy lightning. That day the lightening was generated very close (about $10 \mathrm{~km}$ order) to the ORSOC. These noises could be received by the six antenna pairs, oriented to the six directions. Comparing with the data on the Okayama Local Metrological Office [3], it shows good relations between a flash of lightning and noise pattern in the measurement data.

One more example of a natural noise source is the meteor noise. About the relation between the meteor and FM noise, there are opinions that (1) the meteor noise is reflection of FM radio wave from a station at a great distance from the dust made by the meteors and (2) the meteor itself is a noise origin. Anyway, on the day when the Leonis meteor swarm (November 19, 2001) came down at its maximum as seen in Fig. 9, there was recognized a powerful correlation between the data observed using an antenna oriented to the heaven direction and an emission by lightning of a meteor. Figure 10 shows the result of count number of the pulses over a threshold of $5 \times 10^{-5} \mathrm{~V}$ every 30 minutes. As shown in Fig. 5, a net sampling time is 0.1 second and a sampling interval is 10 seconds. Then, if the continuation of a meteor noise is 0.1 second, realistic number of meteors shall be countered on multiple by 100 for the values of Fig. 10, and also the time of maximum of meteor swarm, coincides with the time of peak in Fig. 10 [4].

As mentioned above, we dealt with the radio wave noise from the galaxy, the flash of lightning and the meteor noise, as well-known electromagnetic noise sources existing in the natural world. On the ground of these facts, the authors consider the data of observation for the 2001 Geiyo earthquake (March 24, 2001). However, at 10:25 AM on April 23rd, we could not record the united data as the apparatus was under testing. Data on April 24th to 29th was recorded under the following conditions:

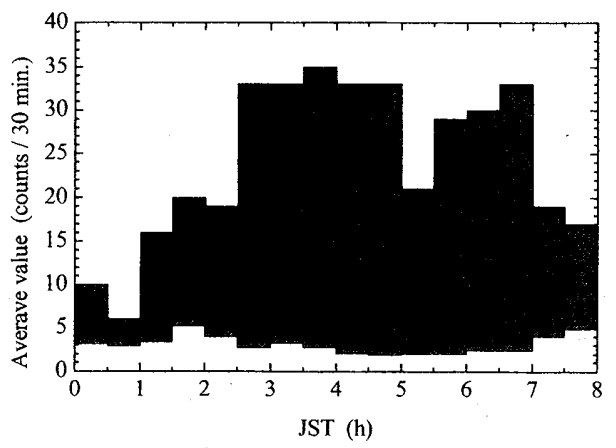

Fig. 10 Number of meteors on November 19, 2001 and other non-event days (November 1 to 18,2001 ). 
- 3/24 0:00 3/25 20:44

Received frequency $94.75 \mathrm{MHz}$

10 elements Yagi-Uda antenna

Output 2 division

An angle of elevation $26^{\circ}$ at Southern direction,

- 3/25 20:44 3/29 24:00

Received frequency $79.75 \mathrm{MHz}$

10 elements Yagi-Uda antenna

Output 12 division

An angle of elevation $26^{\circ}$ at Southern direction.

Fig. 7 shows a noise signal of level $3 \times 10^{-4} \mathrm{~V}$, which occurred at 15:28 of the 2001 Geiyo earthquake. Furthermore, our receiver of the apparatus has caught an alteration of electromagnetic wave noise about nearly 7 hours ahead the earthquake. This alternation kept on for about 13 hours. After 20:44 hours, on March 25th, as the system of input signals from an antenna has modified into 12 parts instead of the earlier 2 parts, then the intensity of the signal level became about one sixth of the input signal. However, we could observe intensity of noise signal over $3 \times 10^{-4} \mathrm{~V}$ kept on record at the previous 2001 Geiyo earthquake, at about the same time of midday on March 26th.

Consequently the authors cannot clearly conclude that the noise wave received at the time of 2001 Geiyo earthquake and the noise wave 7 hours before the start of the quake can be considered as an earthquake precursor. The authors underline the need of additional further study and measurements of the above-discussed natural noise sources.

\section{Conclusion}

The system for earthquake prediction research was completed in the end of March 2001, and is presently running. The system is easy to handle and costs only 660 thousands Yen for the materials cost. Good measurement results of the electromagnetic wave noises caused by thunderbolt were presented in this paper. It seems that the condition of ionosphere has not so strong relation to the transmission of FM radio waves over a long distance range of such $500 \mathrm{~km}$. We suppose that in order to count the number of meteors, the way using electromagnetic wave noise in measurements could be quite useful.

Because the measurements before the Geiyo earthquake were only the pre-earthquake data obtained by means of our system, we cannot definitely classify that data as earthquake prediction one. Only after gathering such more data and further analyses concrete conclusions could be made.

By setting several measurement centers it might be possible to predict not only the epicenter but also the time of occurrence and even the magnitude of earthquakes.

\section{Acknowledgments}

The authors are grateful to the students of Yamamoto's Seismo Electromagnetics Group for their help in measuring the data.

\section{References}

[1] K. Wadatsumi, "What happened? And what is the problem? $\sim$ Last 90 days $\sim$ The Tottori prefecture Seibu earthquake (in Japanese)", ACADEMIA, No.65, 2000.

[2] I. Yamamoto, K. Kuga, T. Okabayashi and T. Azakami, "Bring to the system to completion on the earthquake prediction researches in the region of VHF band (in Japanese)", Technial Report of IEICE, EMCJ 2001-17, 2001.

[3] Measurement data for reference purpose, with the kind of permission of the Okayama Local Meteorological Observatory of the Ministry of Land, Infrastructure and Transportation.

[4] e.g. "Hopping, slipping and jumping in the sly, linger on the brilliant light beam $\sim$ the Leonis meteor swarm additionally observed in Okayama (in Japanese)", the Asahi newspaper, Nov.20, 2001.

[5] T. Fukushima and Y. Ono, "Monitoring of VHF electromagnetic waves using the four quarters antenna system aimed at the detection of precursor of earthquakes", the Japan Earth Planetary Science Joint Meeting 2001, Sj-P003, May 24, 2001. 
[6] T. Yoshida and A. Sato, "Pre-seismic Phenomena and Observations of Naturally Radiated Electromagnetic waves in VHF", Technical Report of IEICE, AP 99-94, SANE99-49, Sept. 1999.

[7] Y. Kushida and R. Kushida, "On the possibility of earthquake forecast by radio observation in the VHF band", RIKEN Review, No. 19, 1-13, 1998.

[8] T. Takano, K. Sakai, A. Yamada and S. Shimakura, "Strategy of observations of seismo-electromagnetic signals at Chiba University", in Seismo Electromagnetics : Lithosphere-Atmosphere Coupling, Ed. by M.Hayakawa and O.A.Molchanov. p.433-435, TERRAPUB Tokyo, 2002.

[9] T. Nagao, "The New View of the Earthquake Prediction Research", Kinmiraisha, Nagoya, 2001.

[10] K. Wadatsumi, "Precursory Quake-Testimony 1519!", Tokyo Shuppan Co. , June 1996.

[11] T. Rikitake, "Earthquake Precursory Phenomena", Tokyo Univ. Pub. Comp., 1986.

(Received February 12, 2002; revised July 30, 2002; accepted August 2, 2002) 